\title{
Article
}

\section{Collaborative healthcare education programmes for continuing professional education in low and middle-income countries: A Best Evidence Medical Education (BEME) systematic review. BEME Guide No. 65}

Hill, Elaine Alais susannna, Gurbutt, Dawne, Makuloluwa, Thamasi, Gordon, Morris, Georgiou, Rachel, Roddam, Hazel, Seneviratne, Sujatha, Byrom, Anna, Pollard, Kerry, Abhayasinghe, Kalpani and Chance-Larsen, Kenneth

Available at http://clok.uclan.ac.uk/39254/

Hill, Elaine Alais susannna ORCID: 0000-0003-4984-9446, Gurbutt, Dawne, Makuloluwa, Thamasi, Gordon, Morris ORCID: 0000-0002-1216-5158, Georgiou, Rachel, Roddam, Hazel ORCID: 0000-0002-0637-1801, Seneviratne, Sujatha, Byrom, Anna, Pollard, Kerry et al (2021) Collaborative healthcare education programmes for continuing professional education in low and middle-income countries: A Best Evidence Medical Education (BEME) systematic review. BEME Guide No. 65. Medical Teacher, 43 (11). pp. 12281241. ISSN 0142-159X

It is advisable to refer to the publisher's version if you intend to cite from the work. https://doi.org/10.1080/0142159X.2021.1962832

For more information about UCLan's research in this area go to http://www.uclan.ac.uk/researchgroups/ and search for < name of research Group>.

For information about Research generally at UCLan please go to http://www.uclan.ac.uk/research/

All outputs in CLoK are protected by Intellectual Property Rights law, including

CLoK

Central Lancashire online Knowledge www.clok.uclan.ac.uk

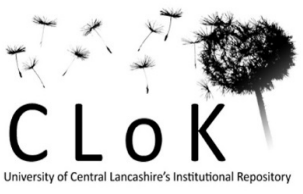




\section{CLoK}

Central Lancashire online Knowledge www.clok.uclan.ac.uk 


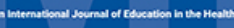

\section{Collaborative healthcare education programmes for continuing professional education in low and middle-income countries: A Best Evidence Medical Education (BEME) systematic review. BEME Guide No. 65}

Elaine Hill, Dawne Gurbutt, Thamasi Makuloluwa, Morris Gordon, Rachel Georgiou, Hazel Roddam, Sujatha Seneviratne, Anna Byrom, Kerry Pollard, Kalpani Abhayasinghe \& Kenneth Chance-Larsen

To cite this article: Elaine Hill, Dawne Gurbutt, Thamasi Makuloluwa, Morris Gordon, Rachel Georgiou, Hazel Roddam, Sujatha Seneviratne, Anna Byrom, Kerry Pollard, Kalpani Abhayasinghe \& Kenneth Chance-Larsen (2021) Collaborative healthcare education programmes for continuing professional education in low and middle-income countries: A Best Evidence Medical Education (BEME) systematic review. BEME Guide No. 65, Medical Teacher, 43:11, 1228-1241, DOI: 10.1080/0142159X.2021.1962832

To link to this article: https://doi.org/10.1080/0142159X.2021.1962832
(c) 2021 The Author(s). Published by Informa UK Limited, trading as Taylor \& Francis Group.

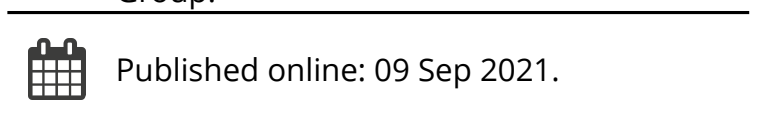

Џlll Article views: 584

\section{View Crossmark data ¿}

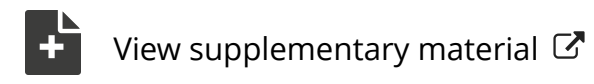

Submit your article to this journal $ک$ 


\title{
Collaborative healthcare education programmes for continuing professional education in low and middle-income countries: A Best Evidence Medical Education (BEME) systematic review. BEME Guide No. 65
}

\author{
Elaine Hill ${ }^{\mathrm{a}}$ (D), Dawne Gurbutt ${ }^{\mathrm{b}}$ (D), Thamasi Makuloluwa ${ }^{\mathrm{c}}$ (D), Morris Gordon ${ }^{\mathrm{d}}$ (D), Rachel Georgiou ${ }^{\mathrm{e}}$ (D), \\ Hazel Roddam $^{a}$ (D), Sujatha Seneviratne ${ }^{f}$ (D) Anna Byrom ${ }^{g}$ (D), Kerry Pollard ${ }^{g}$, Kalpani Abhayasinghe ${ }^{h}$ (D) and \\ Kenneth Chance-Larsen ${ }^{\mathrm{a}}$ \\ ${ }^{\mathrm{a} S}$ School of Sport and Health Sciences, UCLan, Preston, UK; ${ }^{\mathrm{b}}$ Centre for Collaborative Learning, UCLan, Preston, UK; ${ }^{\mathrm{C}}$ Faculty of Medicine, \\ General Sir John Kotelawala Defence University, Ratmalana, Sri Lanka; ${ }^{\mathrm{d} S c h o o l}$ of Medicine, UCLan, Preston, UK; ${ }^{\mathrm{e} S c h o o l}$ of Nursing, \\ UCLan, Preston, UK; ' Department of Nursing and Midwifery, University of Sri Jayewardenepura, Nugegoda, Sri Lanka; ${ }^{9}$ School of \\ Community Health and Midwifery, UCLan, Preston, UK; ${ }^{h}$ Department of Nursing and Midwifery, General Sir John Kotelawala Defence \\ University, Ratmalana, Sri Lanka
}

\begin{abstract}
Background: Large discrepancies exist between standards of healthcare provision in high-income (HICs) and low and middle-income countries (LMICs). The root cause is often financial, resulting in poor infrastructure and under-resourced education and healthcare systems. Continuing professional education (CPE) programmes improve staff knowledge, skills, retention, and practice, but remain costly and rare in low-resource settings. One potential solution involves healthcare education collaborations between institutions in HICs and LMICs to provide culturally appropriate CPE in LMICs. To be effective, educational partnerships must address the challenges arising from differences in cultural norms, language, available technology and organisational structures within collaborating countries. Methods: Seven databases and other sources were systematically searched on 7 July 2020 for relevant studies. Citations, abstracts, and studies were screened and consensus was reached on which to include within the review. 54 studies were assessed regarding the type of educational programme involved, the nature of HIC/LMIC collaboration and quality of the study design.

Results: Studies varied greatly regarding the types and numbers of healthcare professionals involved, pedagogical and delivery methods, and the ways in which collaboration was undertaken. Barriers and enablers of collaboration were identified and discussed. The key findings were: 1 . The methodological quality of reporting in the studies was generally poor. 2 . The way in which HIC/ LMIC healthcare education collaboration is undertaken varies according to many factors, including what is to be delivered, the learner group, the context, and the resources available. 3. Western bias was a major barrier. 4 . The key to developing successful collaborations was the quality, nature, and duration of the relationships between those involved.

Conclusion: This review provides insights into factors that underpin successful HIC/LMIC healthcare CPE collaborations and outlines inequities and quality issues in reporting.
\end{abstract}

\section{KEYWORDS}

Staff development; continuing; general planning; general; low and middle-income countries;

LMIC; collaboration;

healthcare education

\section{Background}

GHWA and WHO (2013) predict that the estimated global deficit of 7.2 million healthcare workers will rise to 18 million by 2030 . Health and healthcare are increasingly acknowledged as global issues, something which has been brought into sharp focus by the 2020 COVID-19 epidemic (Ma et al. 2020). It is ethically unacceptable that huge discrepancies in healthcare practices, provisions, and outcomes exist between LMICs and HICs. LMICS generally lack the infrastructure and resources to address these inequities (Liang and Gong 2017; Cissé 2019), including insufficiency of appropriately trained healthcare staff (Gladding et al. 2018). The Global Nutrition Monitoring Framework (WHO and UNICEF 2017) and the Sustainable Development Goals (UN 2015) have set targets to reduce global health inequities by 2030 and international collaborations between healthcare

\section{Practice points}

- It is important to ensure that CPE programmes for LMICS, which are developed using HIC/LMIC partnerships, are genuinely collaborative and meet the LMIC's needs and resources.

- Developing and nurturing HIC/LMIC contacts over time will maximise the chances of success.

- Ensuring the continuity of $\mathrm{CPE}$, through 'train-thetrainer' programmes and related approaches, is essential.

- Supporting LMIC partners to develop research and publication skills should enable their experiences to be disseminated in the healthcare education literature and reduce Western publication bias.

CONTACT Elaine Hill eashill@uclan.ac.uk $=$ School of Sport and Health Sciences, Brook Building, UCLan, Preston PR1 2HE, UK

(-) Supplemental data for this article can be accessed here.

This article has been corrected with minor changes. These changes do not impact the academic content of the article.

(c) 2021 The Author(s). Published by Informa UK Limited, trading as Taylor \& Francis Group.

This is an Open Access article distributed under the terms of the Creative Commons Attribution License (http://creativecommons.org/licenses/by/4.0/), which permits unrestricted use, distribution, and reproduction in any medium, provided the original work is properly cited. 
- Learning is bi-directional - both HIC and LMIC partners benefit from collaborative educational programmes.

professionals in HICs and LMICs, to develop CPE programmes for LMIC healthcare staff, are an essential component. (TyerViola et al. 2013; Gladding et al. 2018).

Collaborations between HICs and LMICs should involve the exchange of skills and sharing of expertise (Maleka et al. 2019). HICs are generally well resourced and can contribute professional capabilities and specialised resources whilst LMIC partners contribute local clinical and other contextual knowledge (Gladding et al. 2018; Lucas 2019). However, such collaborative working presents many challenges. LMICs may have limited physical and financial resources, including educational and clinical facilities and the necessary staff to manage them (Kim et al. 2015; Ma et al. 2020). The sheer number of staff requiring CPE to improve their skills and practice standards may place further strains on existing services (Macedo et al. 2013; Puchalski Ritchie et al. 2016) and educational approaches developed by well-meaning HICs may fail to take account of local circumstances. For example, several international programmes are only offered in English, or by distance learning, excluding those who lack the necessary language skills, technology, or reliable sources of electricity (Kim et al. 2015; Puchalski Ritchie et al. 2016). These factors combine to limit LMIC staff opportunities for participation in CPE (Willot et al. 2018) which in turn narrows their scope of professional practice, rather than bringing it into line with international standards (Kildea et al. 2012; Puchalsk Ritchie et al. 2016). The situation is made worse by overseas or internal migration in search of better pay, conditions and career opportunities (Saluja et al. 2020) resulting in inequitable workforce distribution (WHO 2013; Gantsho and Wareham 2020).

Well-intentioned programmes devised by HICs may also fail for other Western-centric reasons. For example, some of the current authors have found it difficult to meet the requirements of budget holders when attempting to spend project funds. In a country where institutional email addresses and electronic invoices are the norm, it can be difficult to persuade budget signatories that personal emai accounts and written invoices are legitimate. Power inequities between HICs and LMICs, alongside different agendas and goals, can also leave LMICs potentially open to exploitation by HICs (Gladding et al. 2018; Lucas 2019).

WHO (2013) suggest that effective collaborations result in programmes that are owned and led by the LMIC partner, context-specific, and relevant to the socioeconomic characteristics and needs of the communities they are designed to benefit. DeSantis (1995) Counterpart Concept also proposes that there are four criteria that healthcare education partnerships between HICs and LMICs should meet in order to be truly collaborative:

1. The HIC partners should eventually be able to withdraw, leaving the LMIC partner to successfully run the programme and take ownership of it.

2. Programmes should be designed to meet the specific needs of the LMIC.
3. Sociocultural, political, and economic factors must be considered when planning the programme.

4. The collaboration should develop healthcare partners from the HIC and LMIC to their fullest potential.

Previous reviews have focussed on different aspects of collaborative partnerships between HICs and LMICs. George and Meadows-Oliver (2013) analysed the quality of collaboration by determining which of DeSantis (1995) criteria their included studies met. The review was limited as it did not discuss educational approaches or factors which might enable or prohibit collaboration and it focussed purely on collaborations between nurses. West et al. (2016) examined how midwifery educator capacity was developed in LMICs and found that three key approaches were used. The generalizability of results was limited as some International Confederation of Midwives regions and $\mathrm{WHO}$ were not represented in the publications. Finally, Nishimi and Street (2020) examined the educational delivery approaches used in collaborative programmes and attempted to assess their impact. They concluded that this was difficult as many were in the initial stages of implementation and delivery, and therefore not yet evaluated. The review was limited by focussing solely on nurses and midwives and a lack of clarity over which education related to undergraduate and postgraduate learners.

Given the increasing recognition of global health inequities (Ma et al. 2020), the realisation that HICs and LMICs must work together to find solutions, and growing awareness and uptake of international placements amongst healthcare students, a review of the relevant literature is appropriate. This seeks to answer three distinct questions, which have been framed using Cook et al. (2008) Taxonomy:

- What educational approaches are appropriate when using international collaboration to establish sustainable, culturally appropriate CPE programmes for healthcare professionals in LMICS? (Description)

- What are the barriers and enablers to developing these educational programmes?

- What makes these approaches effective (Justification)?

The findings will be used to determine how healthcare educators in LMICs and HICs should collaborate in order to design and deliver CPE programmes.

\section{Methods}

No single paradigm underpins the review. The questions posed, and the approach to synthesis, arise from constructivism. The transparency and objectivity of positivism influenced the design of data collection tools and processes, but we also acknowledge cultural and sociocultural theory in accepting that the understanding and interpretation of the data will be affected by the cultural lenses of both the authors of the reviewed articles and the authors of this review.

The study protocol was peer-reviewed and published by BEME on 7 October 2019. The types of included studies were broadened on the advice of the reviewers to include reports, surveys, conference papers, posters and proceedings, and descriptive studies. Given the focus of the review, it is crucial to appraise both LMIC and HIC perspectives on collaborations as these may differ. This also necessitated 
minor changes to the data extraction tool. Reporting is structured in accordance with the BEME review checklist (Hammick et al. 2010)

\section{Search strategy}

We used a standardised search strategy (Supplemental appendix 1) and the following databases-Dentistry and Oral Sciences Source, CINAHL Complete, ERIC, Medline fulltext, AMED, PubMed, Open Grey. We also included papers identified through reference lists in included studies and by direct approaches to researchers we knew, or those whose work was included in - or being considered for the review, to enquire about further published or unpublished work. We also hand-searched Association for Medical Education Europe (AMEE) annual meeting proceedings (2013-2019 inclusive) and publications from WHO, United Nations/United Nations High Commission for Refugees, The British Council, Department for International Development, The Tropical Education Trust, Oxfam and Médecins sans Frontières. Finally, some recommendations were made by members of the review team or other academic colleagues.

We contacted authors for further details if it was unclear whether a study met the inclusion criteria, or when we could only obtain abstracts for papers that could potentially be included. If there was no response after two contact attempts, then the study was excluded. Full details of inclusion and exclusion criteria are shown in Table 1 and the definitions we used for 'collaboration', 'CPE', 'healthcare professions/qualified healthcare staff', 'educators' and 'LMIC' are provided in Supplemental appendices 2 and 3. Studies published in any language, country or date were included, alongside all study designs and approaches. Searching for the relevant literature was challenging as we struggled to locate sources of LMIC conference abstracts, posters, and grey literature.

\section{Screening}

Inter-rater reliability was assessed for citation, abstract and full-text screening using Cohen's kappa. The citations and abstracts screening pairs comprised one HIC and one LMIC author. $\mathrm{EH}$ and $\mathrm{KA}$ independently reviewed the citations then $\mathrm{EH}$ and TM independently screened the potentially relevant abstracts, using a checklist (Supplemental appendix 4). Disagreements between the authors were resolved through discussion. Prior to the full-text screening, interrater reliability was checked by all authors coding the same three articles. Full papers were reviewed independently in pairs, wherever possible comprising one HIC and one LMIC author. Disagreements were resolved by discussion or by including a third author.

\section{Data extraction and synthesis}

We used a data extraction form (Supplemental appendix 5) to assess the content of the studies, and the level of HIC/LMIC collaboration, based on guidance from the Best Evidence Medical Education (BEME) Collaboration (Hammick et al. 2010), plus DeSantis (1995); Reid et al. (2005). Where appropriate, study outcomes were related to Kirkpatrick's (1967) framework to assess their effectiveness and could show outcomes at more than one level. Thematic analysis of the barriers to and enablers of - collaboration, which were identified in the studies, was also undertaken to synthesise the evidence.

\section{Evidence synthesis}

We undertook a descriptive synthesis of the included studies using the data gathered on the extraction form. This was used to summarise:

1. Collaboration to develop and deliver education: countries involved, collaborative approaches used, DeSantis (1995) framework, author origins.

2. What was done educationally:

a. Design: educator/learner characteristics, pedagogical approaches, delivery methods and patterns, participant numbers, duration, setting, source.

b. Impact/outcome: Kirkpatrick level, the strength of conclusions

3. Qualitative data relating to barriers/enablers of collaboration identified in the studies: this was synthesised using thematic analysis.

\section{Quality assessment}

It is important to assess the quality and risk of bias in reporting interventions (Gordon et al. 2013), though there is currently no agreed method for doing this (Gordon et al. 2018). We used a Red-Amber-Green (RAG) rating tool (Gordon and Gibbs 2014) (Supplemental appendix 6) to report on the risk of bias in six areas related to the educational intervention or development programme - educational underpinning bias, curriculum bias, setting bias, educational bias, content bias and conclusion bias. Items were judged to be of high quality and low risk of bias (green), unclear quality and risk of bias (yellow) or low quality and high risk of bias related to lack of reporting (red).

Table 1. Inclusion and exclusion criteria.

Inclusion criteria
All study designs and approaches, audits, reports, surveys, conference
paper and proceedings, conference posters, descriptive studies
Collaboration between a HIC and a LMIC
Any healthcare profession, including locally and nationally recognised roles
which exist in LMICs, e.g. trained birth assistants.
Healthcare educators or trainers
Practice or educational settings
CPE activities for qualified healthcare staff
Any date
Any language




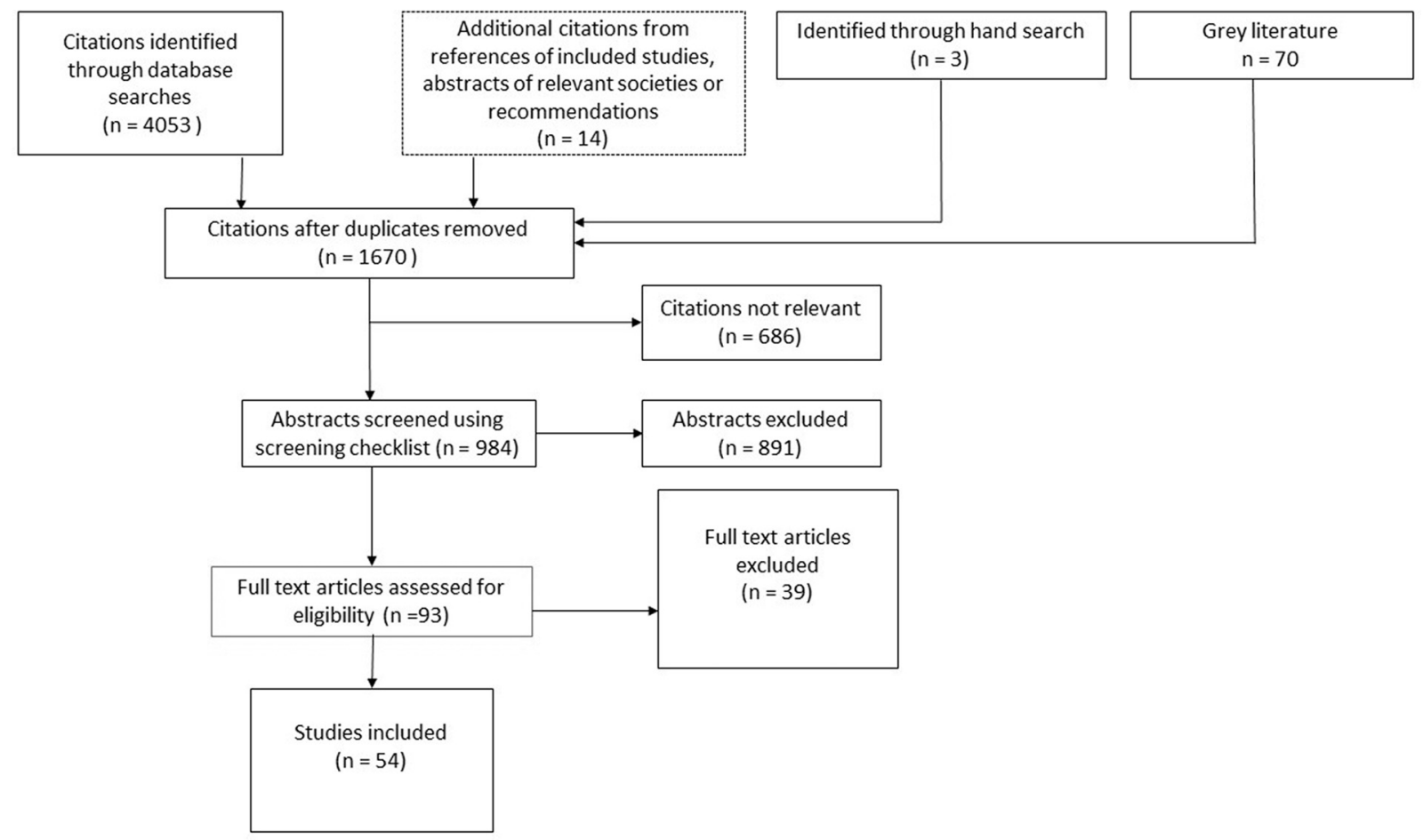

Figure 1. PRISMA flow diagram.

As no area is more important than another, no weighting or overall rank is given; the judgement in each area is provided so readers can assess instances of stronger and weaker reporting. Poor reporting does not necessarily indicate a poor-quality educational development, but it increases the risk that such poor quality may exist. It also limits utility for readers, who may struggle to determine whether the educational development is transferable to their context.

\section{Results}

The search was undertaken on 7 July 2020. This found 4053 citations with a further 87 identified from other sources. Following deduplication 1670 citations remained. 1577 were removed through citation and abstract screening, leaving 93 studies that were identified for full-text screening. 39 were removed following this process leaving 54 which were included in the review; 36 used methods to assess outcomes of the developments and 18 did not. The flow diagram for included studies is shown in Figure 1. The main reasons for exclusion were either no evidence of meeting DeSantis (1995) criteria to evidence collaboration, and therefore not relevant to our area of study, or not contextualizing HIC programmes for delivery in the LMIC, so not relevant to our area of interest. The key data extracted from these papers is summarised in Figures 2 and 3. Full, relevant extraction data, and the list of excluded studies, may be found in Supplementary appendices 7 and 8 respectively. Agreement between citation and abstract screeners using Cohen's kappa statistic was high $(k=.81$ and $k=.89$ respectively); agreement between full-text screeners was fair $(k=.39)$.

Twelve of the studies were quantitative, four were qualitative, and seven mixed methods. The study designs used were surveys (quantitative and qualitative, 10 studies), pre-/ post- (13 studies), interviews (quantitative and qualitative analyses - 9 studies), focus groups (2 studies), observation (1 study), audit (1 study) and analysis of progress reports, presentations and reflective journals (1 study each). Most studies included more than one approach and different combinations were used. In addition, 24 reports, two commentaries, and five studies that focussed on curriculum design were included.

\section{Collaboration as contextualized on DeSantis (1995) framework}

The summary infographic data is provided in Figure 2. DeSantis (1995) provides a recognised structure for assessing the nature of $\mathrm{LMIC} / \mathrm{HIC}$ healthcare education collaborations. Whilst 20 of the studies could only be rated on one of DeSantis' criteria, the majority could be rated on two or more. Most programmes were designed to meet LMIC-specific needs and to take account of the social, political and economic context. In addition, most had sustainable delivery built-in through the inclusion of 'train-the-trainer' or similar elements, to ensure that ownership and responsibility for future delivery lay with the LMIC. The amount of time taken for this to happen would clearly vary according to the nature of the programme. For example, skills and knowledge for delivering a one-day workshop for teaching newborn resuscitation could be established very rapidly (Riley et al. 2019) whilst educating a surgeon to use and disseminate new techniques in clinical practice might take several years (Manske et al. 2017). We found only two studies where the HIC and LMIC partners contributed their unique knowledge and understanding as equals when developing and delivering education (Hojnoski et al. 1998; Binanay et al. 2015).

We also identified seven specific patterns for developing and delivering education to LMIC staff in the studies reviewed. The approach chosen was influenced by the type of education required, the educators and learners and the available resources. 

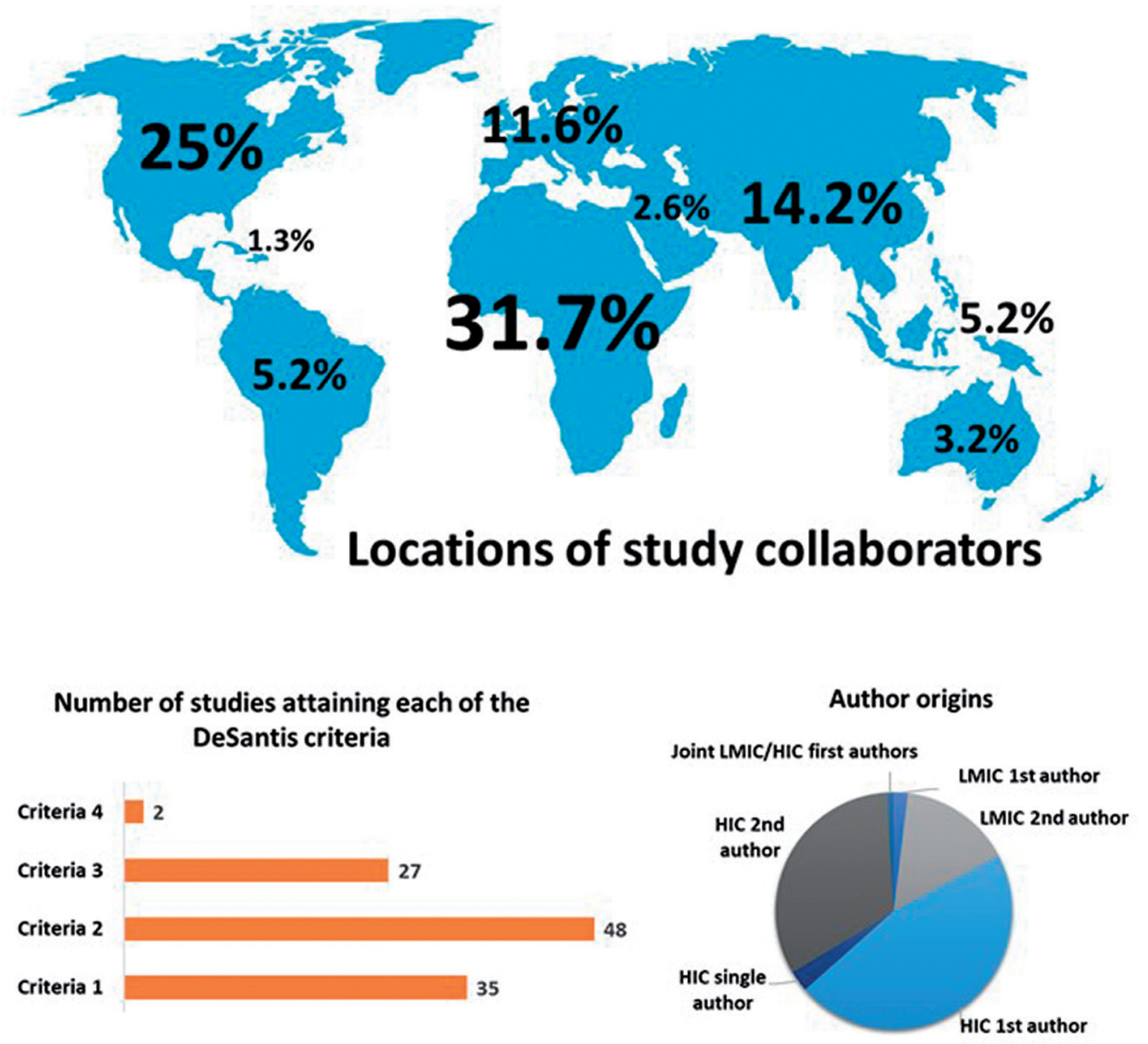

Collaborative approaches used for educating LMIC staff

1.

2. HIC staff educate LMIC staff in the LMIC as part of an international program which is contextualized for the LMIC.

3. HIC clinicians visit the LMIC to work with staff. They educate and develop staff skills.

4. LMIC staff visit the HIC to undertake educational programs or participate in practice e.g. as observers.

5. HIC/LMIC staff exchange programs for teaching and clinical skills development focussing on one or more staff groups in the LMIC.

6. Multi-professional HIC and LMIC staff groups collaborate to develop infrastructure, roles, education and skills. Non-healthcare staff are often included e.g. administrators. For large organisational projects e.g. developing a new coronary care unit.

7. HIC educators include 'train the trainer' approaches, enabling the LMIC to take on educational responsibility from the HIC staff.

Figure 2. Collaborative graphic.

Collaborators came from most geographical regions, with the greatest numbers from Africa, North America, and Asia. The partnership structures varied, sometimes involving one HIC and one LMIC and at other times having more than one HIC or LMIC partner. The overwhelming majority of first and second study authors were from HICs. LMIC partners were significantly under-represented, especially as first authors, a position they occupied in only three studies (Gunathilake et al. 2009; Dapueto et al. 2018; Kisa et al. 2019), sharing joint first authorship with the HIC partner in the latter. None of these studies assessed the impact of interventions. This lack of equitable contribution as authors mirrors the paucity of studies showing equitable contribution by HIC and LMIC partners to the design and delivery of educational programmes, mentioned above.

\section{Details of the intervention or educational programme}

Summary infographic data is provided in Figure 3. Programmes were classified into four categories, according to their origin. Nine were adapted or contextualized from existing university modules or CPE programmes in HICs, 35 were designed specifically for the LMIC, eight were based on existing international initiatives e.g. from $\mathrm{WHO}$ and two 

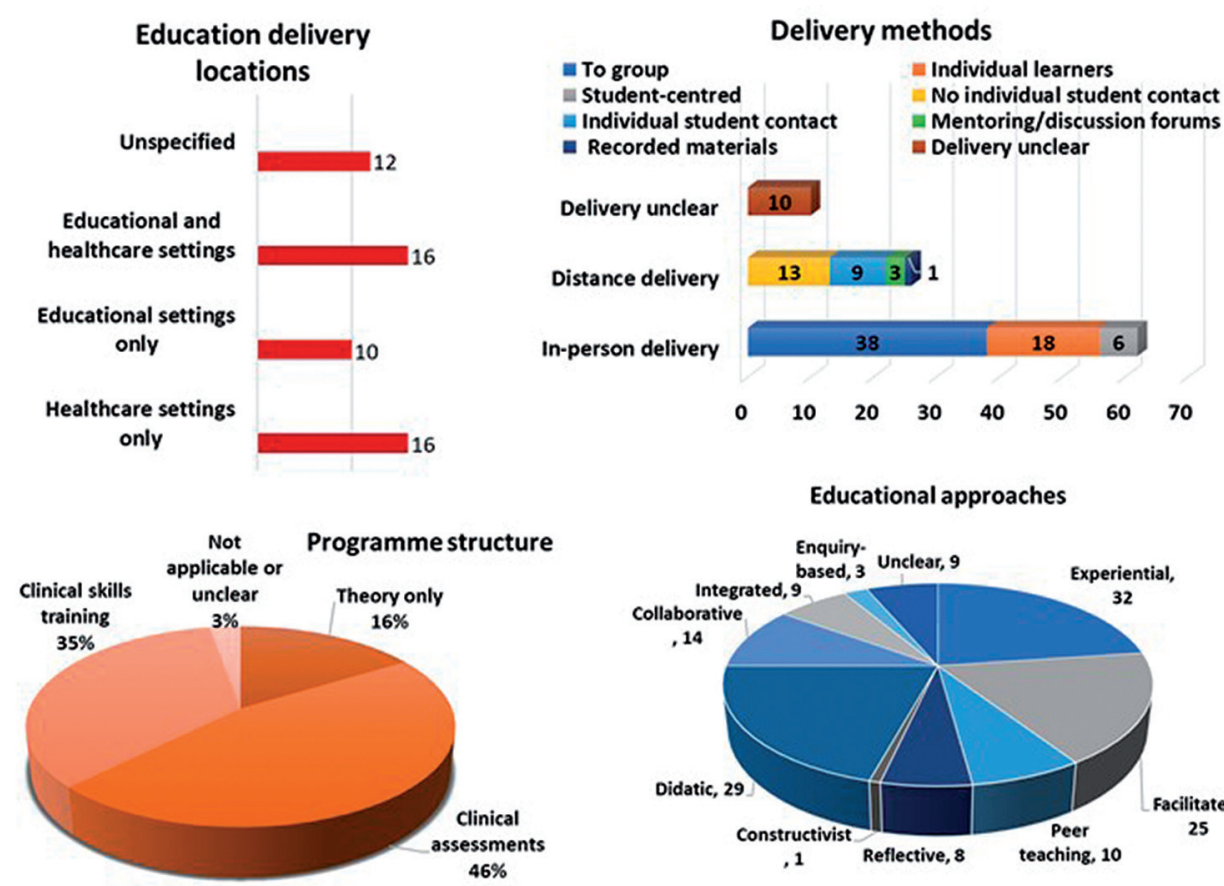

EDUCATOR AND LEARNER PROFESSIONS

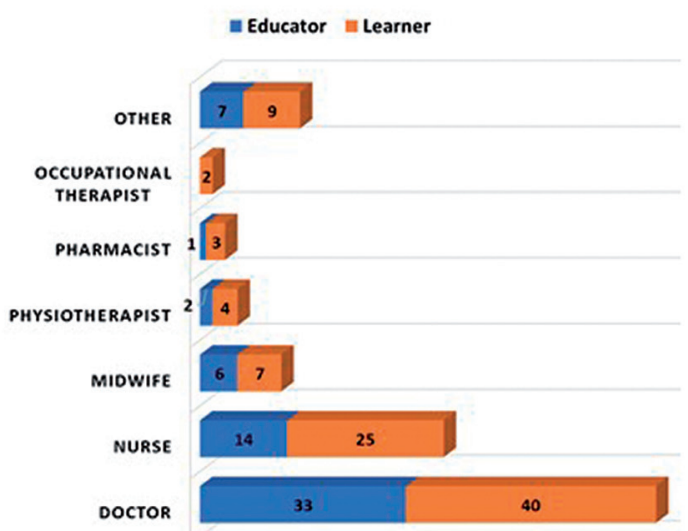

\section{Duration of programme}

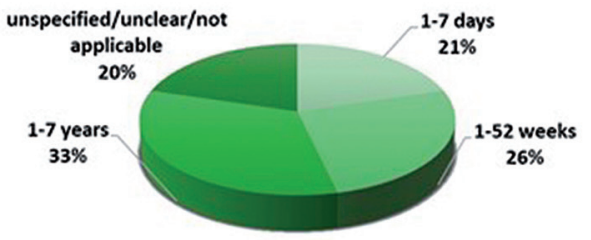

Figure 3. Educational graphic.

were categorized as democratic initiatives. This latter group included the People's University (Heller et al. 2007), a collection of teaching resources donated by the global education community which learners can freely access. It also included work that brought together several disparate organisations providing healthcare education in Uganda to work more collaboratively (Kisa et al. 2019).

Most of the studies focussed on doctors, nurses, and midwives as both educators and learners - professions which are recognized in both HICs and LMICs. However, several studies referred to educational programmes for 'local' professions of trained birth assistants (one study), community healthcare workers (two studies), clinical and medical officers (two studies), Medical Officers of Mental Health (one study) and unspecified local professions (two

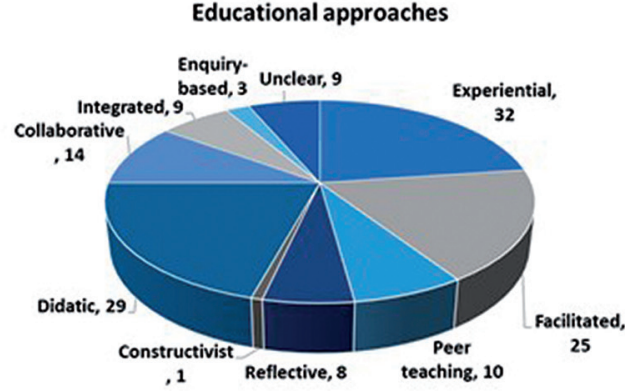

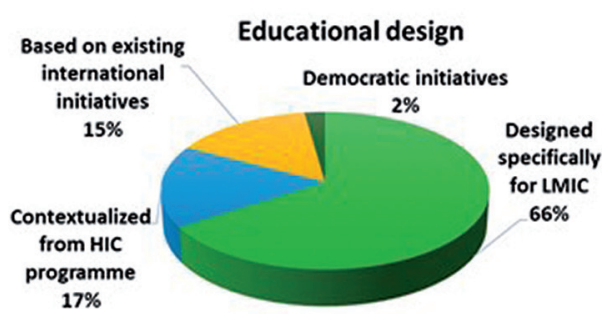

$17 \%$

studies). We found it interesting that two of the studies included administrative staff within the educational programmes, as they were considered integral to the work of the clinical teams, whilst four included non-qualified healthcare staff and students for the same reasons. One study (Riley et al. 2019) included students as educators, alongside qualified staff. In total, ten of the programmes used multi-professional educational teams and 22 were for multi-professional learning groups.

Participant numbers on programmes ranged from 1 to 4600. The median number of participants was 22 with a mode of 13 and a mean of 279 . In total 11,713 participants were mentioned in the course of 42 studies - 12 studies either did not specify participant numbers or focussed on programme designs only. Participant numbers varied according to the programme objectives and duration. 
Barriers: Theme 1:

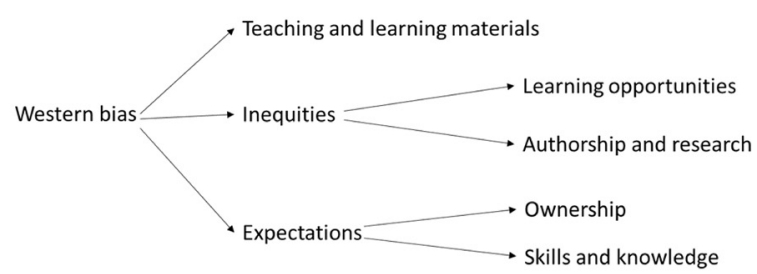

Barriers: Theme 2
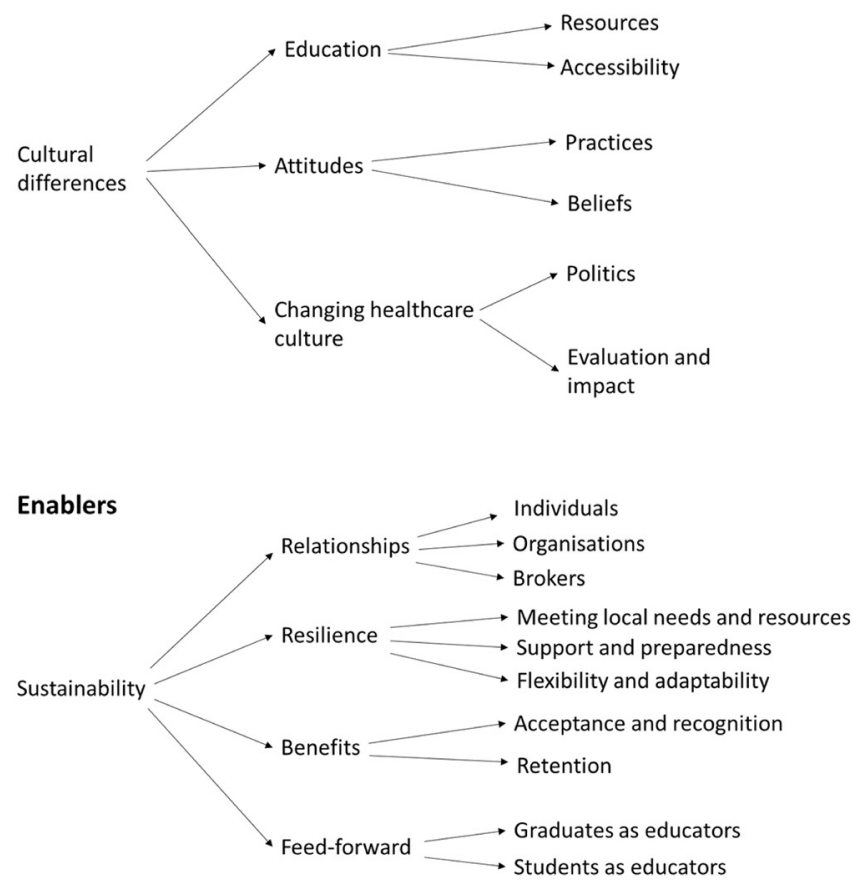

Figure 4. Barriers and enablers themes.

Despite the international nature of the work, and the often-considerable travel involved, in-person delivery to either a group or to individual learners were the most common approaches, which in 16 cases was followed by distance support or delivery. Most programmes included a variety of approaches, which were determined by variables such as the programme length, what was being taught, the learner group, and the delivery location. For example, an 18-month psychiatry Fellowship (Alfonso et al. 2018) included a mixture of in-person delivery to teach theoretical knowledge and clinical skills, which was followed by clinical placements. Distance support was used to follow-up the initial in-person delivery and the educational approaches used were chosen to suit the learning activities. By contrast, a programme which provided a HIC surgeon with a placement in Haiti (Aarabi et al. 2015), to work with LMIC colleagues and enhance their clinical skills, focussed on group teaching and one-to-one support. The delivery locations used were both educational and clinical, often moving between the two depending on what was being taught and/or assessed. Most programmes included clinical skills education or clinical practice and assessment components. Several programmes (e.g. Bell et al. 2014; Foster et al. 2009) involved learners alternating between theory and clinical placements, with the time spent in each varying according to the programme structure. In addition, two programmes provided educational materials on-line and one offered on-line learning to support hospitalbased education.
The duration of educational interventions varied greatly. Longer programmes were more likely to lead to named awards and were generally designed for doctors developing expertise in specialities, such as surgery, paediatrics, oncology or anaesthesia. Such programmes lasted between two and seven years, though in one study the education was open-ended. Named/outcome assessment awards were also available for nurses, midwives and physiotherapists, with programmes lasting between one and three years. In some cases, programmes for doctors and other staff complemented one another, such as the three-year cardiology award for medical staff which was supported by a threeyear programme for ECG technicians and a six-month award for cardiology nurses (Binanay et al. 2015). This study also included training for administrative staff working on the cardiology unit. Shorter CPE programmes targeted very specific skills and issues e.g. paediatric resuscitation. In 11 papers the duration of the educational delivery was unclear.

\section{Impact and outcome assessment}

Only 36 studies of the included studies can be described using Kirkpatrick's framework. 12 studies were at level 1, indicating that the participants reacted to the educational intervention. Nine studies were at level $2 a$ indicating a change in attitudes and 25 studies achieved level $2 \mathrm{~b}$, indicating an increase in participants' knowledge and skills as a result of education. 12 studies achieved level 3, showing a change in participants' behaviours following the educational intervention. Finally, three studies had outcomes at level $4 a$, as there were organisational changes and four studies showed findings at level $4 \mathrm{~b}$ as patients benefitted as a result of staff education.

Regarding the strength of conclusion, six studies were scored at $1 / 5$, indicating that no clear conclusions could be drawn from the results. Six studies scored $2 / 5$ as the results were ambiguous and sixteen studies scored $3 / 5$ as the conclusions could probably be based on the results. Eight studies scored $4 / 5$ as the results were considered to be clear and likely to be true. Finally, none of the studies were felt to have unequivocal results. 18 of the studies could not be rated as they did not assess the outcomes of the intervention or development.

\section{Methodological quality of reporting}

The risk of reporting bias was evaluated using the RAG tool for the 36 studies which assessed the outcomes of the educational intervention or development. Only one study (Peter et al. 2016) was deemed of high quality (green) regarding theoretical underpinning, with the majority rated as poor (red). Educational approaches were described well (green) in only two studies (Holm and Burkhartzmeyer 2015; Peter et al. 2016). In both cases the studies demonstrated clear links between theory and practice. Curriculum, setting, content, and conclusions were generally poorly described.

The reports focussed principally on the collaborative aspects of educational design and delivery, rather than assessing the outcomes of educational interventions or development, and consequently, most could not be evaluated using the RAG tool. Those which could be were generally considered at high risk of bias (red) on all aspects of quality 
as they provided insufficient evidence to support their results. However, two reports (Bell et al. 2014; Tuggle et al. 2017) provided some general information about curriculum (yellow) and three reports provided general information on educational approaches (yellow) (Conway et al. 2017; Tuggle et al. 2017; Manske et al. 2017); the latter also reported clearly (green) on the setting and learner characteristics.

The relatively large proportion of qualitative and mixed methods studies that were included also helped to provide more holistic insights into collaborative working; these are less likely to be apparent in quantitative papers, which focus principally on the design and outcomes of interventions.

\section{Thematic analysis}

\section{Barriers}

Two themes were identified from the studies, each with three subthemes (Figure 4).

\section{Theme 1: Western bias}

\section{i. Teaching and learning materials}

Most textbooks, care models, and educational programmes are developed in HICs and therefore relevant to the pathology and available treatment approaches in them. Pathology and treatments in LMICs may differ.

\section{ii. Inequities}

Learning opportunities: LMIC learners have less opportunity to travel to HICs for educational purposes and when they do, they may be limited in clinical areas to observerships and their qualifications may not be recognized. Some knowledge gained may also lack relevance as the clinical approaches used may be unavailable in their home countries. LMIC learners may also have less opportunity to attend international conferences as these are disproportionately expensive, regarding both money and time.

Authorship and research: LMIC staff often lack opportunities to engage in research or develop skills in writing for publication; this can preclude publication, particularly in Western journals.

\section{iii. Expectations}

Ownership: HICs often want to 'own' projects and their results and expect exclusive collaboration with a particular LMIC partner. By contrast, some LMICs may wish to share materials and work with more than one HIC.

Skills and knowledge: HICs may make incorrect assumptions regarding LMIC staff knowledge and skills as this may differ to what is normal in their home country, often due to differences in available resources.

\section{Theme 2: Cultural differences}

\section{i. Education}

Resources: Lack of resources is a major barrier to developing and delivering education. Funding in LMICs may be very limited or unavailable and teaching facilities more basic than those in HICs. Technology may be dated and unreliable due to the vagaries of electricity supplies, geography, and weather. It may also fail to meet HIC expectations regarding security, internet access, bandwidth, software, and availability of individual email addresses and on-line materials, all of which may reduce options for teaching and learning delivery. Healthcare equipment may be similarly dated and supplies intermittent. Finally, restricted availability of printed textbooks and photocopying facilities may limit learners' access to information and teaching materials whilst scenarios that are not contextualized, or delivered using unfamiliar approaches, may limit engagement, applicability, and understanding.

Accessibility: Education should be offered locally in LMICs to enable access for reasons relating to both gender roles/culture and available transport, especially in remote, rural areas. HIC educators may misinterpret absence or lateness as a result of these factors and become frustrated with what they perceive as lack of engagement, rather than recognising that their learners require support. Finally, many programmes are in English and not always translated into local languages, unwittingly excluding some potential learners.

\section{ii. Attitudes}

Practices: It is considered normal for HIC partners to travel to the LMIC country. This may impact negatively on the time and resources available for collaboration due to jet lag, costs and absence from their home employment.

Beliefs: HIC and LMIC partners may have very different concepts about health and wellbeing which impact upon interventions to treat and manage them.

\section{iii. Changing healthcare culture}

Politics: Change is unlikely to occur in LMICs without the support of political and professional hierarchies as new skills and professions must be acknowledged and supported by relevant approval, and possibly legislation and accreditation. Corruption can also be problematical in some settings. HICs may struggle to navigate these problems successfully.

Evaluation and impact: Changes in practice and improved patient outcomes are often difficult to assess due to a lack of appropriate evaluation tools and proper record keeping. In addition, existing management and evaluation systems frequently provide no information regarding cost-effectiveness.

\section{Enablers}

One theme was identified from the studies; this had four subthemes.

\section{Sustainability}

\section{i. Relationships}

Individual: The quality and duration of the relationships between collaborators are key to success.

Organisations: Buy-in from those in power in organisations such as universities, government ministries, and healthcare organisations is needed to for educational programmes to be promoted and approved. 
Brokers: Cultural brokers or sympathetic partners who can negotiate across cultural divides, and with those in positions of power or influence, may be vital to the success of projects.

\section{ii. Resilience}

Meeting local needs and resources: Programmes must meet identified local needs and be culturally appropriate in order to have value and purpose. Delivering programmes locally and outside of staff work hours may also increase the likelihood of attendance. Free study materials should be provided, translated into local languages, and where necessary interpreters should be included in teaching sessions. Delivery methods must be appropriate to the available resources, including staff, equipment, and facilities and relevant educational approaches should be deployed.

Support and preparation: LMIC partners and local staff delivering programmes should be properly prepared, both for their educational role and for managing any issues which may arise.

Flexibility and adaptability: Programmes will be more resilient and sustainable if creative solutions can be found to address potential threats or difficulties

\section{iii. Benefits}

Acceptance and recognition: For programmes to flourish and have a lasting impact new practices and qualifications must be accepted by clinicians and employers. This may present challenges, especially in remote, regional facilities, as changes may be perceived as a threat by senior practitioners.

Retention: If staff can use their skills, and be employed in new roles, they have greater job satisfaction. They are consequently more likely to continue working locally than to migrate, either within or outside of their home country.

\section{iv. Feed forward}

Graduates as educators: Programmes become sustainable as previous graduates take on their delivery.

Students as educators: Including HIC students as educators may promote interest in international healthcare issues and prompt them to become involved in developing $\mathrm{HIC} /$ LMIC healthcare education programmes in the future.

\section{Discussion}

PubMed data alone shows that the number of publications on international healthcare education collaborations has grown rapidly since 2014, which coincides with growing awareness of global health inequities, increased recognition of the work undertaken to address this, and greater opportunities for undertaking international collaborations.

\section{Methodological quality of reporting}

The included studies focussed on either the educational intervention and its impact or the collaborative aspects of programmes. The finding that methodological quality is generally poorly reported in medical education studies (Cook et al. 2007; Cook et al. 2011) was echoed in this review. Only one study included a clear description of its underpinning educational framework (Peter et al. 2016) and just two studies clearly described the educational approaches used (Holm and Burkhartzmeyer 2015; Peter et al. 2016). Curriculum was poorly described in most of the studies, limiting the potential for these to inform future developments.

Discussion of interventions was generally limited in the studies which focussed specifically on collaboration, resulting in a high risk of reporting bias due to a lack of evidence. However, these studies often contained excellent, detailed information about how collaborations were undertaken and the barriers and enablers which they reveal. In some cases, high risks of reporting bias occurred due to the nature of the intervention.

\section{Designing and delivering education}

The approaches used to deliver healthcare education, and create effective learning environments, varied according to what was being delivered, to whom and the desired outcomes. The approaches in turn determined the choice of delivery methods and location, participant numbers, collaborative approach, delivery pattern, program origin, duration, educational approach and whether the programme involved multi-professional educators and/or learners. These options may be conceptualized as a toolbox from which educators can select appropriately when designing interventions. However, what they can choose will also be limited by the resources available to them. Consequently, all programmes in the reviewed studies use unique, individual approaches to effectively address the needs of learners, whilst simultaneously falling within the resource boundaries and being culturally appropriate. A variety of adaptations and creative approaches were used to ensure accessibility and engagement of learners and sustainable delivery.

Contextualization of HIC programmes was effective in situations where there was a single, correct approach to manage a pathophysiological problem, for example, resuscitating newborn babies (Riley et al. 2019) or surgery for specific medical conditions (e.g. Cameron et al. 2010; Gordon et al. 2006). However, this could pose considerable challenges and stress for HIC educators to ensure safe and effective patient outcomes whilst being limited by dated treatment options and equipment (Rogers 2016). Preparation and support for HIC staff undertaking such education were included in several studies (e.g. Blignault et al. 2012; Burton et al. 2019).

\section{Changing practice}

Unfortunately, new knowledge and skills did not always translate into changes in practice. Of the studies reviewed only 12 resulted in changes in behaviour, four reported benefits to patients and three resulted in changes to institutional practices. Possible reasons include lack of resources or equipment and resistance from senior clinicians, who may feel undermined by new knowledge and practices (Fagan et al. 2016). The likelihood of acceptance increases if programmes are endorsed or accredited by recognised HIC bodies (Cameron et al. 2010) or by patients/public sharing 


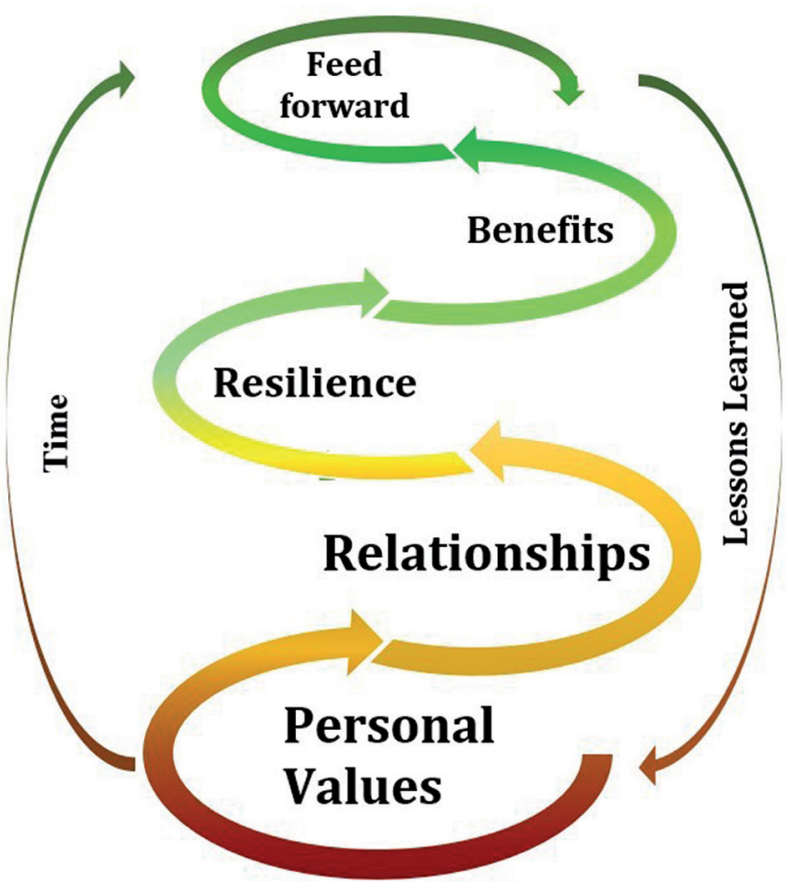

Figure 5. The collaborative model.

their positive experiences (Gunathilake et al. 2009; Vargas et al. 2012). Restrictions on applying new learning contribute to reduced job satisfaction and the migration of LMIC clinicians overseas (Deckelbaum et al. 2014), so it is essential these issues are addressed to enable staff retention.

\section{Western bias}

This was identified as a major barrier.

\section{Educational bias}

Educational resources and care models developed in HICs may lack relevance in LMICs as they focus on HIC pathology and treatment approaches (Ndura 2004; Aarabi et al. 2015; Carter et al. 2016), which may explain why most interventions in the studies were developed specifically for the LMIC. Unconscious bias within individuals (Hall et al. 2015; Fitzgerald and Hurst 2017; Rimmer 2017) and institutions (Pritlove et al. 2019), combined with not understanding that LMIC learners may lack expected knowledge and skills due to an absence of facilities and opportunities (Ventres and Wilson 2015), may also limit LMIC learners to observerships in HIC clinical areas. Whilst several of the studies reviewed provided learning opportunities for LMIC staff in the HIC partner country, only three enabled them to participate more fully in clinical practice (Gunathilake et al. 2009; Blignault et al. 2010, 2012), with the first study involving a clinical placement for participating doctors in the UK National Health Service. One study (Gordon et al. 2006) resolved the problem by arranging placements in a better-resourced LMIC.

Unconscious bias or HIC/LMIC power differentials may also explain why LMIC and HIC partners contributed as equals to the design of programmes in only two of the studies reviewed (Hojnoski et al. 1998; Binanay et al. 2015). Whilst most programmes met LMIC-specific needs, were appropriate to the social, political and economic context and were designed to be sustainable through LMIC ownership, they may ultimately have a greater susceptibility to failure if the LMIC partner's unique knowledge and understanding do not underpin them, which has ethical as well as practical implications.

\section{Publishing bias}

We largely failed to access sources of LMIC-based literature in the databases available to us and LMIC authors were grossly under-represented in the studies reviewed. The LMIC voice is frequently absent from international, highimpact publications (Patel and Sumathipala 2001; Mulimani 2019) due to long-recognized reviewer and publishing biases (Ross et al. 2006; Harris, Macinko, et al. 2017; Harris, Marti, et al. 2017). The situation is further complicated by the rejection of LMIC articles due to a lack of perceived impact, poor methodological quality and weak writing style. Whilst this may appear objectively legitimate, it fails to recognise that the reasons relate to inequity and lack of capacity (Tyrer 2005). The dominance of English as the language of research, alongside bias towards publishing studies with positive results (Wells and Laurence 2020), may also present structural barriers to LMIC participation (Kelaher et al. 2016).

Consequently, an estimated $90 \%$ of the published literature represents the needs and concerns of the richest $10 \%$ of the global population, primarily based in HICs, a situation which has been likened to institutional racism (Tyrer 2005). Whilst low-resource countries bear the greatest burden of disease (Mulimani 2019) and ill-health, opportunities to contribute their unique insights and experience to the pool of healthcare knowledge are severely limited. However, without their contribution our wider understanding is flawed (Tyrer 2005), with subsequent impacts on professional practice and treatment options (Weber 2019). Similarly, knowledge of healthcare education will be incomplete if the LMIC voice is missing from the literature.

This situation is unlikely to change unless LMIC capacity is increased through changes to funding (Kelaher et al. 2016), providing opportunities to participate in research and support to develop academic writing skills (Mash et al. 2014; Franzen et al. 2017). Only two studies reviewed (Cancedda et al. 2014; Cameron et al. 2017) specifically mentioned increasing the research capacity of LMIC partners to address these issues.

\section{The current review}

As most authors of reviewed studies were from HICs the barriers and enablers identified in their work are predominantly viewed through a Western lens. Whilst the same issues may also affect LMIC partners and learners, their observations on the same issues may be subtly different. Using the Cultural Diversity Lens (UNESCO 2011) could offer one solution to evaluating and amending educational programmes, whilst simultaneously supporting cultural diversity. Similar issues are likely to have arisen within the review team as the cultural lenses of the UK and Sri Lanka authors will differ, but the greater collective experience of the UK authors may have unconsciously influenced the interpretation of the data. 


\section{Relationships}

The key to successful collaboration seems largely determined by the quality and nature of the relationships between the HIC and LMIC partners. Collaborations frequently built upon existing relationships which took many years, and successive projects, to establish. These were often multi-professional and based upon shared values of openness, trust and equity, cultural humility, reflexivity, and strong personal commitments to challenge injustices and relieve suffering (Aniekwe et al. 2012; Binanay et al. 2015; McKimm et al. 2019).

This is consistent with cultural and sociocultural theory. Culture has been likened to an onion (Hofstede and Hofstede 2005) where the innermost layer of cultural values determines the appearance of the surrounding layers of rituals and traditions, heroes, and the outermost, visible layer of cultural artefacts (such as food and architecture). Whilst people may learn reasonably quickly to interact appropriately with the tangible aspects of another culture, establishing meaningful relationships, and deeper understanding, involves accessing and comprehending its hidden, underlying values. This learning occurs gradually as partners interact and internalize one another's cultural ways of thinking and knowing (Vygotsky 1986), which may help explain the importance of 'cultural brokers' for translating cultural nuances whilst collaborators develop the skills to do this themselves.

\section{Collaborative model}

Data synthesised from the thematic analysis indicates that successful collaboration may be conceptualized as a spiral (Figure 5). This contains five progressive stages which collaborators may need to navigate many times before they reach the pinnacle of programmes to which the HIC and LMIC partners contribute as equals (DeSantis 1995) and where learners can apply their skills in practice, patients benefit and there are changes to healthcare infrastructure (Kirkpatrick 1967).

\section{Personal values}

Anecdotal evidence suggests that these may be a necessary pre-condition upon which the enablers rest and which nurture and sustain collaborative relationships.

\section{Relationships}

These need to develop slowly between the HIC and LMIC partners, building gradually as projects increase in scope and complexity.

\section{Resilience}

The issues encountered in collaborative developments may be positive (such as huge demands for the programme) or negative (such as resistance to change from local hospitals or lack of time to undertake the work). Developing programmes slowly allows testing and adjustments to occur at each stage and enables collaborators to develop confidence and creative solutions to problems. This also develops resilience in both people and programmes.

\section{Benefits}

As programme graduates begin to apply new skills in practice, they benefit from greater job satisfaction, their employer benefits from improved staff retention and patients benefit from improved outcomes.

\section{Feed forward}

The process of collaboration and learning must continue, both for existing collaborators and into the next generation. LMIC programme graduates may sustain the process through undertaking 'train-the-trainer' programmes or by disseminating their skills and knowledge through teaching junior staff as their level of seniority increases. Including HIC learners as educators on programmes may increase their awareness of global health issues and inspire them to collaborate with LMIC partners in the future.

Negotiating the spiral takes time and repetition, and progress may vary in speed and direction. An analogy may be drawn between this process and the board game Snakes and Ladders where sometimes players progress rapidly by climbing the ladders and bypassing sections of the board whilst on other occasions, they encounter a snake and slide back several stages. The model may explain why relatively few programmes demonstrated achievements that reached the 'pinnacle', and these generally took longer to develop.

\section{Limitations}

This review has several limitations. Western bias is highly likely to have favoured the publication of studies led by HICs and the journal databases available will have determined which of these we could access. In addition, publication bias towards studies with positive results may have prevented those which had negative, yet useful, findings from being made known. Accessing relevant grey literature was problematic so potentially relevant unpublished studies were not located. The focus of the review itself, and the search terms used, may also have failed to capture relevant research, and due to word limitations, several potentially interesting investigative avenues had to be either excluded or not pursued in depth. The inability to determine whether some authors were from LMICs, but based in HICs, also places limitations on the work. Finally, it is likely that Western bias has affected the review itself, despite taking steps to try and mitigate this. Most authors are from the UK, the work is written for a HIC journal, and the lens through which data has been viewed and interpreted is shaped largely by Western research paradigms.

\section{Implications for teaching}

Sociocultural learning theory (Vygotsky 1986) suggests that the earlier students begin to interact with other cultures, the more readily they will internalize and understand their ways of thinking, which is important for generating interest, engagement, and potential long-term partnerships.

Changes to healthcare curricula could allow international healthcare to be introduced early in undergraduate programmes to generate interest and engagement from learners, which could be developed further as their studies progress. Where available, modern technology can 
support shared learning and collaboration across continents and some inspiring global classroom initiatives are already being undertaken (McKimm et al. 2019) which allow LMIC and HIC students to learn together in virtual space. Changes to curricula could also enable learning undertaken through HIC/LMIC student collaborations to be incorporated into course assessments. The existing HIC international curricula could also be refocussed to support more LMIC/HIC learner exchanges.

HIC/LMIC collaborations should also focus on developing research capacity and academic writing skills for LMIC partners as without their unique knowledge and insights, our understanding of global health and disease remains incomplete. Where appropriate, distance approaches should be considered by collaborators to reduce the carbon footprint from travel; this would enable the travelling time to be used for collaborative work instead.

Finally, it is essential to remember that knowledge flows in both directions. One obvious example is the growing focus on mindfulness education for HIC healthcare students to improve mental wellbeing and reduce burnout, a technique that is drawn principally from Buddhist meditation (Chung et al. 2018). The more students and practitioners from different cultures work and learn together and from one another, the greater the benefits for both them and their patients.

\section{Implications for research}

There are implications for both educational, leadership, and sociocultural research. Studies focussing on changes to educational curricula may enable understanding of how best to foster the next generation of collaborators. Research into leadership and change management in LMICs could support programme graduates to apply new skills in practice and improve health outcomes and staff retention in LMICs. It is also crucial to find effective ways of developing research and publication skills for LMIC authors so they may contribute to the global healthcare community. Whilst this review has provided some insights into what underpins effective collaboration, further research is needed to better understand the processes, and enable better intercultural working and sharing of knowledge.

\section{Conclusions}

This review has highlighted the importance of $\mathrm{LMIC} / \mathrm{HIC}$ collaborations for developing CPE programmes for LMICs. Sadly, it has also revealed that inequities between HIC and LMIC learners persist and the methodological quality of reporting in research on collaborations is generally poor. Positively, the review has provided some insights into the mechanisms which may underpin effective collaborations, which may benefit those involved. This is of crucial importance given the challenges of increasing the global healthcare workforce.

\section{Acknowledgment}

Authors thank Clipart from Openclipart.org.

\section{Disclosure statement}

$\mathrm{EH}, \mathrm{DG}$ and MG are BEME BICC members.

\section{Funding}

Open access funding support from the Research Institute on Global Health and Wellbeing, UCLan, UK.

\section{Notes on contributors}

Elaine Hill, MSc, BSC, PGCE, Dip HE (RN), ODP, MBPPs, is a senior lecturer at the University of Central Lancashire, UK, with interests in international healthcare education, climate change and health, interprofessional learning and social justice.

Dawne Gurbutt, PhD, MA, BSc, PGCE, is professor of collaborative education at UCLan, UK with interests in inter-professional education, collaborative learning, creativity in the curriculum and service user engagement.

Thamasi Makuloluwa, MBBS, MD(Anaesthesiology), FRCA(Lond), is professor of anaesthesia at General Sir John Kotelawala Defence University (KDU), Sri Lanka with research interests in pain medicine and medical education.

Morris Gordon, $\mathrm{MBChB}$, $\mathrm{PhD}, \mathrm{MMed}$, is Cochrane co-ordinating editor, chair of the BEME executive committee, and a professor of evidence synthesis and systematic review, UCLan, UK.

Rachel Georgiou, MSc, BSc, RGN, is a senior research fellow and development manager for UCLan's Research Institute for Global Health and Wellbeing, with a specialist interest in global health and stroke.

Hazel Roddam, $\mathrm{PhD}, \mathrm{MA}, \mathrm{BSc}$, is an experienced researcher in allied health practice. She has an international reputation as an advocate and mentor for clinical academic roles in allied health services.

Sujatha Seneviratne, Mphil, MN, BSCN, BSc, RN, is a senior lecturer in nursing at the University of Sri Jayawardenepura, Sri Lanka with research interests in clinical nursing and nursing education.

Anna Byrom, PhD, PGCE, BSc, RM, NTFS, is a senior lecturer in midwifery at UCLan, UK and an active member of the THRIVE Research Centre. She is the director of All4Maternity and publisher of The Practising Midwife and The Student Midwife journals.

Kerry Pollard, MSc PG Cert HE RM RN, is a senior lecturer at UCLan with a special interest in improving outcomes for women and infants through the high-quality education of health care professionals.

Kalpani Abhayasinghe, $\mathrm{PhD}, \mathrm{BScN}, \mathrm{RN}$, is a senior lecturer in nursing at KDU, Sri Lanka with research interests in qualitative methodology, community care, mental health nursing and health promotion

Kenneth Chance-Larsen, MSc, BSc, PGCE, is a senior lecturer in physiotherapy and MSC (Pre-reg) Physiotherapy course leader at UCLan, UK, with a special interest in curriculum development and musculoskeletal health.

\section{ORCID}

Elaine Hill (ID) http://orcid.org/0000-0003-4984-9446

Dawne Gurbutt (ID http://orcid.org/0000-0002-6916-3276

Thamasi Makuloluwa (D) http://orcid.org/0000-0002-2530-7821

Morris Gordon (D) http://orcid.org/0000-0002-1216-5158

Rachel Georgiou (ID http://orcid.org/0000-0002-0920-0602

Hazel Roddam (ID http://orcid.org/0000-0002-0637-1801

Sujatha Seneviratne (ID) http://orcid.org/0000-0001-7108-7199

Anna Byrom (D) http://orcid.org/0000-0003-4408-8545

Kalpani Abhayasinghe (iD http://orcid.org/0000-0002-9180-7023

Kenneth Chance-Larsen (D) http://orcid.org/0000-0002-7619-4054

\section{References}

Aarabi S, Smithers C, Louis Fils M-M, Godson J-L, Pierre J-H, Mukherjee J, Meara J, Farmer P. 2015. Global surgery fellowship: a model for surgical care and education in resource-poor countries. J Pediatr Surg. 50(10):1772-1775. 
Alfonso CA, Michael MC, Elvira SD, Zakaria H, Kalayasiri R, Adlan ASA Moinalghorabaei M, Lukman PR, San'ati M, Duchonova K, et al. 2018. Innovative educational initiatives to train psychodynamic psychiatrists in underserved areas of the world. Psychiatr Clin North Am. 41(2):305-318.

Aniekwe CC, Hayman R, Mdee A, Akuni J, Lall P, Stevens D. 2012. Academic-NGO collaboration in international development research: a reflection on the issues. Working paper. Development Studies Association. https://www.intrac.org/wpcms/wp-content/uploads/ 2016/09/Academic-NGO-Collaboration-in-InternationalDevelopment_September-2012.pdf

Bell S, Oteng R, Redman R, Lapham J, Bam V, Dzomecku V, Yakubu J, Tagoe N, Donkor P. 2014. Development of an emergency nursing training curriculum in Ghana. Int Emerg Nurs. 22(4):202-207.

Binanay C, Akwanalo CO, Aruasa W, Barasa FA, Corey GR, Crowe S, Esamai F, Einterz R, Foster MC, Gardner A, et al. 2015. Building sustainable capacity for cardiovascular care at a public hospital in western Kenya. J Am Coll Cardiol. 66(22):2550-2560.

Blignault I, Manicavasagar V, Chang O, Parker G. 2010. Description and evaluation of a project to improve the identification and management of mood disorders in developing countries. Australas Psychiatry. 18(5):460-464.

Blignault I, Manicavasagar V, Parker G, Wijeratne C, Sumathipala A 2012. Building capacity in identification and management of mood disorders: an Australia-Sri Lanka collaboration. Australas Psychiatry. 20(1):53-56.

Burton ZA, Ayele Y, McDonald P. 2019. Establishing a sustainable anaesthetic education programme at Jimma University Medical Centre, Ethiopia. Anaesth Intensive Care. 47(4):334-342.

Cameron B, Rambaran M, Sharma DP, Taylor RH. 2010. The development of postgraduate surgical training in Guyana. Can J Surg. 53(1) $11-16$.

Cameron L, Johnstone JC, Sparman A, Nelin LD, Singh NC, Hunter A. 2017. Guyana's paediatric training program: a global health partnership for medical education. Can Med Educ J. 8(2):e11-e17.

Cancedda C, Farmer PE, Kyamanywa P, Riviello R, Rhatigan J, Wagner CM, Ngabo F, Anatole M, Drobac PC, Mpunga T, et al. 2014. Enhancing formal educational and in-service training programs in rural Rwanda: a partnership among the public sector, a nongovernmental organization, and academia. Acad Med. 89(8):1117-1124.

Carter M, Levin NW, Carter CS, Callegari J. 2016. The Sustainable Kidney Care Foundation's contribution to the improvement of AKI management in developing countries using peritoneal dialysis. Clin Nephrol. 86(13):78-S83.

Chung AS, Felber R, Han E, Mathew T, Rebillot K, Likourezos K. 2018. A targeted mindfulness curriculum for medical students during their emergency medicine clerkship experience. West J Emerg Med. 19(4):762-766

Cissé G. 2019. Food-borne and water-borne diseases under climate change in low- and middle-income countries: further efforts needed for reducing environmental health exposure risks. Acta Trop. 194: 181-188.

Conway DJ, Coughlin R, Caldwell A, Shearer D. 2017. The Institute for Global Orthopedics and Traumatology: a model for academic collaboration in orthopaedic surgery. Front Public Health. 5:Article 146.

Cook DA, Beckman TJ, Bordage G. 2007. Quality of reporting of experimental studies in medical education: a systematic review. Med Educ. 41(8):737-745.

Cook DA, Bordage G, Schmidt HG. 2008. Description, justification and clarification: a framework for classifying the purpose of research in medical education. Med Educ. 42(2):128-133.

Cook DA, Levinson AJ, Garside S. 2011. Method and reporting quality in health professions education research: a systematic review. Med Educ. 45(3):227-238

Dapueto JJ, Viera M, Samenow C, Swiggart WH, Steiger J. 2018. A tale of two countries: innovation and collaboration aimed at changing the culture of medicine in Uruguay. HEC Forum. 30(4):329-339.

Deckelbaum DL, Gosselin-Tardif A, Ntakiyiruta G, Liberman S, Vassiliou M, Rwamasirabo E, Gasakure E, Fata P, Khwaja K, Razek T, et al. 2014. An innovative paradigm for surgical education programs in resource-limited settings. Can J Surg. 57(5):298-299.

DeSantis L. 1995. A model for counterparts in international nursing. Int J Nurs Stud. 32(2):198-209.

Fagan J, Aswani J, Otiti J, Mushamba V, Liyombo E, Woodson G, Weed D, Zender C, Mannion K, Netterville J, et al. 2016. Educationa workshops with graduates of the University of Cape Town Karl Storz Head and Neck Surgery Fellowship Program: a model for collaboration in outreach to developing countries. Springerplus. 5(1): 1652.

Fitzgerald C, Hurst S. 2017. Implicit bias in healthcare professionals: a systematic review. BMC Med Ethics. 18(1):19.

Foster K, Usher K, Gadai S, Taukei R. 2009. There is no health without mental health: implementing the first mental health nursing postgraduate program in Fiji. Contemp Nurse. 32(1-2):179-186.

Franzen S, Chandler C, Lan T. 2017. Health research capacity development in low and middle income countries: reality or rhetoric? A systematic meta-narrative review of the qualitative literature. BMJ Open. 7(1):e012332.

Gantsho L, Wareham C. 2020. Medical cosmopolitanism: the global extension of justice in healthcare practice. Dev World Bioeth. 00: 1-8. DOI:10.1111/dewb.12278.

George EK, Meadows-Oliver M. 2013. Searching for collaboration in international nursing partnerships: a literature review. Int Nurs Rev. 60(1):31-36.

Gladding S, McGann P, Summer A, Russ C, Uwemedimo O, Aguilar M Chakraborty R, Moore M, Lieh-Lai M, Opoka R, et al. 2018. The collaborative role of North American departments of pediatrics in global child health. Pediatrics. 142(1):e20172966.

Global Heath Workforce Alliance [GHWA], World Health Organisation [WHO]. 2013. Universal truth: no health without a workforce: third Global Forum on Human Resources for Health Report. Geneva: WHO.

Gordon G, Vongvichit E, Hansana V, Torjesen K. 2006. A model for improving physician performance in developing countries: a threeyear postgraduate training program in Laos. Acad Med. 81(4): 399-404.

Gordon M, Darbyshire D, Saifuddin A, Vimalesvaran K. 2013. Limitations of poster presentations reporting educational innovations at a major international medical education conference. Med Educ Online. 18(1):1-4.

Gordon M, Gibbs T. 2014. STORIES statement: publication standards for healthcare education evidence synthesis. BMC Med. 12(1):143.

Gordon M, Hill E, Stojan J, Daniel M. 2018. Educational interventions to improve handover in health care: an updated systematic review. Acad Med. 93(8):1234-1244.

Gunathilake W, Idampitiya CA, Siriwardana A, Lenora D, Thomson GA Fernando DJS. 2009. An international partnership to implement innovative systems of health care delivery for diabetes in a developing country. Pract Diab Int. 26(4):145-147.

Hall W, Chapman M, Lee K, Merino Y, Thomas T, Payne K, Eng E, Day S, Coyne-Beasley T. 2015. Implicit racial/ethnic bias among health care professionals and its influence on health care outcomes: a systematic review. Am J Public Health. 105(12):e60-e76.

Hammick M, Dornan T, Steinert Y. 2010. Conducting a best evidence systematic review. Part 1: from idea to data coding. BEME guide no. 13. Med Teach. 32(1):3-15.

Harris M, Macinko J, Jimenez G, Mullachery P. 2017. Measuring the bias against low-income country research: an implicit association test. Global Health. 13(1):9.

Harris M, Marti J, Watt H, Bhatti Y, Macinko J, Darzi A. 2017. Explicit bias toward high-income-country research: a randomized, blinded, crossover experiment of English clinicians. Health Aff. 36(11) 1997-2004.

Heller RF, Chongsuvivatwong V, Hailegeorgios S, Dada J, Torun P, Madhok R, Sandars J. 2007. Capacity-building for public health: http://peoples-uni.org. Bull World Health Organ. 85(12):930-934.

Hofstede G, Hofstede GJ. 2005. Cultures and organizations: software of the mind. 2nd ed. New York: McGraw-Hill.

Hojnoski JA, Ciottone GR, Aghababian RV. 1998. International developments of emergency medical systems: educational techniques for the future. Eur J Emerg Med. 5:23-27.

Holm MR, Burkhartzmeyer HL. 2015. Implementation of a phased medical educational approach in a developing country. Glob Health Action. 8:29882

Kelaher M, Ng L, Knight K, Rahadi A. 2016. Equity in global health research in the new millennium: trends in first-authorship for randomized controlled trials among low- and middle-income country researchers 1990-2013. Int J Epidemiol. 45(6):2174-2183. 
Kildea S, Larsson M, Govind S. 2012. A review of midwifery in Mongolia utilising the 'Strengthening Midwifery Toolkit'. Women Birth. 25(4):166-173.

Kim D-H, Yoon HB, Sung M, Yoo D-M, Hwang J, Kim EJ, Lee S, Shin JS 2015. Evaluation of an international faculty development program for developing countries in Asia: the Seoul Intensive Course for Medical Educators. BMC Med Educ. 15:224.

Kirkpatrick DL. 1967. Evaluation of training. In: Craig RL, Bittel LR, editors. Training and development handbook. New York (NY): McGrawHill.

Kisa P, Grabski DF, Ozgediz D, Ajiko M, Aspide R, Baird R, Barker G, Birabwa-Male D, Blair G, Cameron B, et al. 2019. Unifying children's surgery and anesthesia stakeholders across institutions and clinica disciplines: challenges and solutions from Uganda. World J Surg. 43(6):1435-1449.

Liang L, Gong P. 2017. Climate change and human infectious diseases: a synthesis of research findings from global and spatio-temporal perspectives. Environ Int. 103:99-108.

Lucas AO. 2019. International collaboration in health research. Bull World Health Organ. 83(7):1.

Ma X, Vervoort D, Reddy CL, Park KB, Makasa E. 2020. Emergency and essential surgical healthcare services during COVID-19 in low- and middle-income countries: a perspective. Int J Surg. 79:43-46.

Macedo CR, Macedo EC, Torloni MR, Atallah ÁN. 2013. Evaluation of a national e-based healthcare course via teleconference in a developing country. J Eval Clin Pract. 19(4):713-719.

Maleka NM, Currie P, Schneider H. 2019. Research collaboration on community health worker programmes in low-income countries: an analysis of authorship teams and networks. Glob Health Action. 12(1):1606570.

Manske MCB, Rios Roque JJ, Zelaya GR, James MA. 2017. Pediatric Hand surgery training in Nicaragua: a sustainable model of surgical education in a resource-poor environment. Front Public Health. 5:75.

Mash R, de Villiers M, Moodley K, Nachega J. 2014. Guiding the development of family medicine training in Africa through collaboration with the Medical Education Partnership Initiative. Acad Med. 89(8 Suppl):S73-S77

McKimm J, McLean M, Gibbs T, Pawlowicz E. 2019. Sharing stories about medical education in difficult circumstances: conceptualizing issues, strategies, and solutions. Med Teach. 41(1):83-90.

Mulimani P. 2019. Publication bias towards western populations harms humanity. Nat Hum Behav. 3(10):1026-1027.

Ndura E. 2004. ESL and cultural bias: an analysis of elementary through high school textbooks in the western United States of America. Lang Cult Curric. 17(2):143-153.

Nishimi K, Street NM. 2020. Nursing education partnerships between western high-income universities and non-governmental agencies and low-income local agencies: a scoping review of the literature. J Prof Nurs. 36(3):147-157.

Organisation for Economic Co-operation and Development [OECD] 2018. DAC list of ODA recipients: effective for reporting on 2018 2019 and 2020 flows; [accessed 2020 Nov 28]. https://www.oecd. org/dac/financing-sustainable-development/development-financestandards/DAC_List_ODA_Recipients2018to2020_flows_En.pdf.

Patel V, Sumathipala A. 2001. International representation in psychiatric literature: survey of six leading journals. $\mathrm{Br} J$ Psychiatry. 178(108):406-409.

Peter NA, Pandit H, Le G, Nduhiu M, Moro E, Lavy C. 2016. Delivering a sustainable trauma management training programme tailored for low-resource settings in east, central and southern African countries using a cascading course model. Injury. 47(5):1128-1134.
Pritlove C, Juando-Prats C, Ala-Leppilampi K, Parsons JA. 2019. The good, the bad, and the ugly of implicit bias. Lancet. 393(10171): 502-504.

Puchalski Ritchie LM, Khan S, Moore JE, Timmings C, van Lettow $M$, Vogel JP, Khan DN, Mbaruku G, Mrisho M, Mugerwa K, et al. 2016. Low- and middle-income countries face many common barriers to implementation of maternal health evidence products. J Clin Epidemiol. 76:229-237.

Reid D, Price EG, Windish DM. 2005. Challenges in systematic reviews of educational intervention studies. Ann Intern Med. 142(12): 1080-1089.

Riley C, Spies LA, Prater L, Garner SL. 2019. Improving neonatal outcomes through global professional development. Adv Neonatal Care. 19(1):56-64.

Rimmer A. 2017. Unconscious bias must be tackled to reduce worry about overseas trained doctors, says BAPIO. BMJ. 357:j1881.

Rogers D. 2016. Which educational interventions improve healthcare professionals' resilience? Med Teach. 38(12):1236-1241.

Ross JS, Gross CP, Desai MM, Hong Y, Grant AO, Daniels SR, Hachinski VC, Gibbons RJ, Gardner TJ, Krumholz HM. 2006. Effect of blinded peer review on abstract acceptance. JAMA. 295(14):1675-1680.

Saluja S, Rudolfson N, Massenburg B, Meara J, Shrime N. 2020. The impact of physician migration on mortality in low and middleincome countries: an economic modelling study. BMJ Glob Health. 5(1):e001535.

Tuggle D, Rasnake N, Day Y. 2017. Improving trauma care in Africa. J Trauma Nurs. 24(4):258-260.

Tyrer P. 2005. Combating editorial racism in psychiatric publications. Br J Psychiatry. 186(1):1-3.

Tyer-Viola LA, Timmreck E, Bhavani G. 2013. Implementation of a continuing education model for nurses in Bangladesh. J Contin Educ Nurs. 44(10):470-476.

United Nations [UN]. 2015. Sustainable Development Goals; [accessed 2020 Nov 28]. https://www.un.org/sustainabledevelopment/development-agenda/.

United Nations Educational, Scientific and Cultural Organization [UNESCO]. 2011. The Cultural Diversity Lens: a practical tool to integrate culture in development. Paris: UNESCO.

Vargas G, Price R, Sergelen O, Lkhagvabayar B, Batcholuun P, Enkhamagalan T. 2012. A successful model for laparoscopic training in Mongolia. Int Surg. 97(4):363-371.

Ventres W, Wilson C. 2015. Beyond ethical and curricular guidelines in global health: attitudinal development on international servicelearning trips. BMC Med Educ. 15:65-68.

Vygotsky LS. 1986. Thought and language. Cambridge (MA): MIT Press. Weber E. 2019. Publication bias begins at home. Emerg Med J. 36(9): 518-519.

Wells B, Lawrence D. 2020. Potential publication bias in chiropractic and spinal manipulation research listed on clinicaltrials.gov. J Can Chiropr Assoc. 64(1):82-87.

West F, Homer C, Dawson A. 2016. Building midwifery educator capacity in teaching in low and lower-middle income countries. A review of the literature. Midwifery. 33:12-23.

Willot C, Sakashita R, Genden-Jamts E, Yoshino Y. 2018. Distance learning for maternal and child health nurses and midwives in Mongolia: a qualitative evaluation. Int Nurs Rev. 65(4):577-585.

World Health Organisation [WHO]. 2013. Transforming and scaling up health professionals' education and training: WHO guidelines 2013 Geneva: WHO.

World Health Organisation/United Nations Children's Fund [WHO/ UNICEF]. 2017. Global nutrition monitoring framework: operational guidance for tracking progress in meeting targets for 2025. Geneva: WHO. 